\title{
Adsorption of 1,10-Phenanthroline-2,9-dithiol on Gold and Silicon Surfaces
}

\author{
Petr Sokolov, ${ }^{* \dagger}{ }^{\dagger}$ Victor Demidov, ${ }^{\ddagger}$ Lidia Vedeneeva, ${ }^{\S}$ and Nina Kasyanenko ${ }^{\dagger}$ \\ ${ }^{\dagger}$ Saint Petersburg State University, 7-9 Universitetskaya nab., Saint-Petersburg 199034, Russia \\ "Company "Pro-Brite”, Sofyiskaya, 93, Saint-Petersburg 192289, Russia \\ ${ }^{\S}$ Perm National Research Polytechnic University, Khimikov, 10 Perm region, Berezniki 618400, Russia
}

ABSTRACT: Derivatives of 1,10-phenanthroline are used in bio- and chemosensors as well as in supramolecular chemistry. A reliable method of fixation onto gold, silicon, and other surfaces is necessary to solve some problems in these fields and will provide new application opportunities. In this study, adsorption of a sulfur-containing 1,10-phenanthroline derivative (PhenSH) onto gold and silicon surfaces was examined using atomic force microcsopy, surface plasmon resonance (SPR), and $\mathrm{X}$-ray photoelectron spectroscopy techniques and compared to that of a sulfur-free derivative. We report PhenSH-Au bond formation with most likely orthogonal orientation with respect to the surface. The adsorption process proceeds by forming a layer of molecules that are not firmly bound to the surface which then form a covalent bond. The compound is sterically accessible to reagents in the solution, such as metal ions, which will allow using its coordinating properties to create SPR and surface-enhanced Raman spectroscopy-based chemosensors and to attach phenanthroline-based supramolecular nanostructures to nanoparticles or nanodevices.

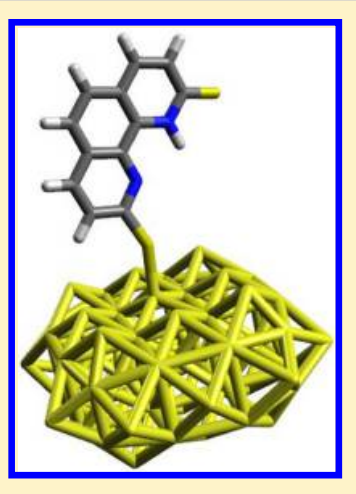

\section{INTRODUCTION}

Derivatives of 1,10-phenanthroline have unique properties ${ }^{1}$ which allow the creation of anticancer drugs, ${ }^{2,3}$ chemosensors, ${ }^{4-6}$ biosensors, ${ }^{7-11}$ and self-organizing controlled supramolecular structures, ${ }^{12-16}$ which can be used in medicine and nanotechnology. The fixation of 1,10-phenanthroline derivatives from a solution onto silicon, mica, glass, gold, and other surfaces is necessary to solve some problems in these fields. The adsorption of 1,10-phenanthroline has been extensively studied with surface-enhanced Raman spectroscopy ${ }^{17-19}$ (SERS) and scanning tunneling microscopy ${ }^{20-22}$ (STM) methods. Investigations have mainly focused on the self-organization of phenanthroline layers on solid surfaces. The nature of the substituents and steric properties of 1,10-phenanthroline derivatives are of great importance during interaction with the substrate. In particular, the presence of reactive mercapto groups (thiol groups) in the compound promotes the formation of strong bonds with the gold surface, which can be used in formulating various nanostructures. ${ }^{23,24}$

The ability of phenanthroline derivatives to bind with different macromolecules can be used in nanotechnology to fix the supramolecular structures on the surface, to modify the surfaces of various devices when creating bio- and chemosensors based on 1,10-phenanthroline derivatives, and for coupling of compounds with metal nanoparticles. Thus, study of the adsorption and binding of 1,10-phenanthroline derivatives to gold and silicon surfaces is of particular interest and could pave the way for the further use of these compounds in new technologies.

\section{EXPERIMENTAL METHODS}

Polycrystalline yellow powder 1,10-phenanthroline-2,9-dithiol (1,10-dihydro-1,10-phenanthroline-2,9-dithione), termed
PhenSH, was synthesized according to the procedure described previously. ${ }^{25}$ Analysis showed a content of C 59.5\%, H 3.9\%, and $\mathrm{N} 12.1 \%$. For $\mathrm{C}_{12} \mathrm{H}_{8} \mathrm{~N}_{2} \mathrm{~S}_{2}$, the following was calculated: $\mathrm{C} 59.0 \%$, $\mathrm{H} 3.3 \%$, and $\mathrm{N} 11.5 \%$. During the study, we also used commercial formulation of 2,9-dimethyl-1,10-phenanthroline hemihydrate, $\mathrm{C}_{14} \mathrm{H}_{12} \mathrm{~N}_{2} \cdot 0.5 \mathrm{H}_{2} \mathrm{O}$, neocuproine, white polycrystalline powder (hereinafter Phen $\mathrm{CH}_{3}$, Acros Organics, 99\%, CAS 484-11-7, New Jersey, US). The structures of compounds are depicted in Figure 1.

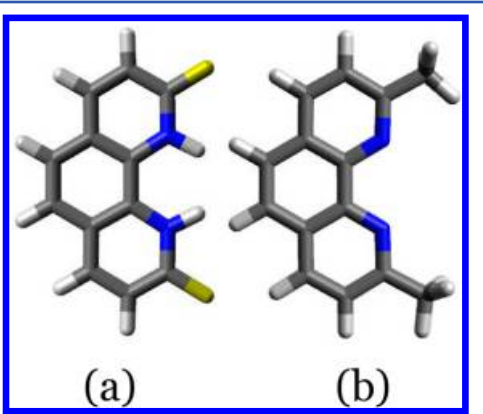

Figure 1. Structures of PhenSH (in a 1(H),10(H)-phenanthroline-2,9dithione form) (a) and $\mathrm{PhenCH}_{3}$ (b). Nitrogen is marked with blue, sulfur with yellow. The structures were visualized in the Avogadro 2 0.7.2 program and optimized using potentials of the universal force field (UFF).

Received: June 11, 2015

Revised: October 1, 2015 